\title{
Reconstructing the velocity dispersion profiles from the line-of-sight kinematic data in disc galaxies
}

\author{
A. A. Marchuk ${ }^{1 \star}$ and N. Y. Sotnikova ${ }^{1,2}$ \\ ${ }^{1}$ St. Petersburg State University, Universitetskij pr. 28, 198504 St. Petersburg, Stary Peterhof, Russia \\ ${ }^{2}$ Isaac Newton Institute of Chile, St. Petersburg Branch, Russia
}

Accepted XXX. Received YYY; in original form ZZZ

\begin{abstract}
We present a modification of the method for reconstructing the stellar velocity ellipsoid (SVE) in disc galaxies. Our version does not need any parametrization of the velocity dispersion profiles and uses only one assumption that the ratio $\sigma_{z} / \sigma_{R}$ remains constant along the profile or along several pieces of the profile. The method was tested on two galaxies from the sample of other authors and for the first time was applied to three lenticular galaxies NGC 1167, NGC 3245 and NGC 4150 as well as to one Sab galaxy NGC 338. We found that for galaxies with a high inclination $\left(i>55-60^{\circ}\right)$ it is difficult or rather impossible to extract the information about SVE while for galaxies at an intermediate inclination the procedure of extracting is successful. For NGC 1167 we managed to reconstruct SVE, provided that the value of $\sigma_{z} / \sigma_{R}$ is piecewise constant. We found $\sigma_{z} / \sigma_{R}=0.7$ for the inner parts of the disc and $\sigma_{z} / \sigma_{R}=0.3$ for the outskirts. We also obtained a rigid constrain on the value of the radial velocity dispersion $\sigma_{R}$ for highly inclined galaxies and tested the result using the asymmetric drift equation, provided that the gas rotation curve is available.
\end{abstract}

Key words: galaxies: fundamental parameters - galaxies: kinematics and dynamics

\section{INTRODUCTION}

One of the most challenging problems of galactic dynamics is reconstructing the distribution function (DF) of a certain galaxy from its limited observational data including surface photometry and long-slit or two-dimensional field spectroscopy. Various techniques have been invented last several years in order to solve this problem.

The most developed scheme is based on Schwarzschild linear programming method (Schwarzschild 1979). It uses a library of orbits in a given potential to reproduce the structure and kinematics of a stellar system, mainly triaxial models (see, e.g. Capuzzo-Dolcetta et al. 2007; van den Bosch et al. 2008 and references therein). There is a particle-based extension of the Schwarzscild' method (made-to-measure method). It works by adjusting individual particle weights as the model evolves, until the $N$-particle system reproduces a set of target constraints (see e.g. Syer \& Tremaine 1996; de Lorenzi et al. 2007, 2008). A new iterative method for constructing equilibrium phase models of stellar systems (Rodionov \& Sotnikova 2006; Rodionov et al. 2009) has been tested on galaxies with imitated observational data and has shown its flexibility (Rodionov et al. 2009).

All these algorithms need a detailed mass model of a target galaxy, so nearly all dynamical models have been applied

^ E-mail: a.marchuk@spbu.ru (AAM) for one-component system, mainly for elliptical galaxies. There are still no reliable complete phase models of observed spiral galaxies that are multicomponent systems with a substantial mass contribution of invisible matter - dark haloes. One obvious exception is the Milky Way with a detailed phase model (Bovy \& Rix 2013).

In many cases we do not need a DF to judge about dynamic status of a stellar disc since it is sufficient to know the velocity dispersion profiles, mainly $\sigma_{R}(R)$ and $\sigma_{z}(R)$. The radial velocity dispersion profile $\sigma_{R}$ gives $Q=\sigma_{R} / \sigma_{R}^{\text {cr }}$ (Toomre 1964), where $\sigma_{R}^{\text {cr }}=3.36 G \Sigma_{\mathrm{s}} / \kappa$ for an infinitely thin stellar disc, $\kappa$ is the epicyclic frequency and $\Sigma_{\mathrm{s}}$ is the disc surface density. The $Q$-parameter shows the level of disc heating and stability against perturbations in a disc plane. The ratio $\sigma_{z} / \sigma_{R}$ describes the shape of the stellar velocity ellipsoid (SVE) and tells about the relaxation in a disc and its dynamical history.

The $\sigma_{z} / \sigma_{R}$ ratio for stars in the solar neighbourhood is $0.53 \pm$ 0.07 (Dehnen \& Binney 1998). Until recently, there were no data available for external galaxies about the $\sigma_{z} / \sigma_{R}$ ratio. Even for the nearby galaxy M31 individual stellar measurements give only the line-of-sight profile of the velocity dispersion (Dorman et al. 2015). Observations of external galaxies have been concentrated on systems that are close either to face-on or to edge-on cases (e.g. van der Kruit \& Freeman 1986; Bottema 1993; Kregel et al. 2005). Face-on systems provide direct information only about one component of random velocities $-\sigma_{z}(R)$. For edge-on systems we 
do not obtain $\sigma_{R}(R)$ or $\sigma_{\varphi}(R)$ directly because of the integration of the DF along the line-of-sight. The profile $\sigma_{R}(R)$ can be obtained implicitly via the asymmetric drift equation that describes the equilibrium in a plane of a rotating stellar disc. The procedure needs the usage of the gas and stellar velocity curves. A major concern is that the integration along the line-of-sight can have a dramatic effect on the derived rotation curve. This effect leads to a marked discrepancy between the rotation curve and the circular velocity curve especially in the inner part of a galaxy (Zasov \& Khoperskov 2003; Stepanova \& Volkov 2013). As a result the scheme needs the restoration of the rotation curve (Di Teodoro \& Fraternali 2015) and more or less robust parametrization of $\sigma_{R}(R)$ (Kregel et al. 2005).

All three moments of the random velocity distribution in a stellar disc can be found for a galaxy at an intermediate inclination. Long-slit data from a variety of position angles and large-area integral field units provide information about the line-of-sight velocity dispersion $\sigma_{\text {los }}$ at different points in a galaxy. All three components $\sigma_{R}, \sigma_{\varphi}$ and $\sigma_{z}$ contribute to $\sigma_{\text {los }}$ depending on the azimuthal angle and inclination. There are two ways to derive these components. One way is to construct a set of maximum-mass marginally stable discs ( $N$-body models). Then the line-of-sight velocity dispersion profiles can be constructed and compared with observational data to choose the most reliable model (Bottema \& Gerritsen 1997; Zasov et al. 2008, 2012). Another way is to pick out $\sigma_{R}, \sigma_{\varphi}$ and $\sigma_{z}$ directly from $\sigma_{\text {los }}$ along the major and minor axes, provided the epicycle approximation that connects $\sigma_{R}, \sigma_{\varphi}$ and the rotational velocity of stars $\bar{v}_{\varphi}$ (Gerssen et al. 1997, 2000; Shapiro et al. 2003; Gerssen \& Shapiro Griffin 2012; Verheijen et al. 2004; Noordermeer et al. 2008).

To derive the velocity dispersion profiles directly exponential approximations for both radial and vertical velocity dispersion components are usually assumed. The most conventional ansatz of a such parametric approach is $\sigma_{z}^{2}(R) \propto \Sigma_{\mathrm{s}}(R)$ (the assumption is valid for an isothermal layer with a constant thickness, Spitzer 1942) and $\sigma_{z} / \sigma_{R}=$ const throughout a disc (Verheijen et al. 2004). There are numerical bases that stellar discs are vertically isothermal (e.g. Rodionov \& Sotnikova 2006). For an exponential disc with a scalelength $h$ the assumption $\sigma_{z}^{2}(R) \propto \Sigma_{\mathrm{s}}(R)$ results in $\sigma_{z}(R) \propto \exp (-R / 2 h)$ and this agrees fairy well with observational data (van der Kruit \& Searle 1981). The assumption $\sigma_{z} / \sigma_{R}=$ const leads to the exponential profile for $\sigma_{R}$ with a scalelength $2 h$ (see, e.g. Martinsson et al. 2013). The above assumption has no clear evidences and is not supported by $\mathrm{N}$-body simulations (see, e.g. Minchev et al. 2012) but is commonly accepted in theoretical analysis.

Gerssen et al. (1997, 2000), Shapiro et al. (2003), Gerssen \& Shapiro Griffin (2012) used a more free parametrization of the velocity dispersion profiles. They let the scale length for $\sigma_{R}(R)$ and $\sigma_{z}(R)$ be not equal to $2 h$ and obtained that it was a factor 3-5 larger than the disc scale length $h$. The velocity dispersion profiles derived by Noordermeer et al. (2008) from spectroscopic data along the major and minor axes got also very shallow. These results contradict with the approximation of isothermal layers and force to revise the procedure of extracting the velocity dispersion profiles from the line-of-sight kinematics.

Instead of using any parametrization for the velocity dispersion profiles like Gerssen et al. (1997, 2000), Shapiro et al. (2003), Gerssen \& Shapiro Griffin (2012), in Sil'Chenko et al. (2011) authors followed another approach. They used an equation containing velocity dispersions and difference between the stellar rotation curve and the local circular speed (the so-called asymmetric drift equation, see equation 4). If one has data for both gas and stellar rotation curves then this equation might be solved iteratively. Sil'Chenko et al. (2011) applied this approach to the early-type disc galaxy NGC 7217. Retrieved independently from the major and minor line-of-sight data two profiles of $\sigma_{z}(R)$ are in good agreement and derived $\sigma_{R}(R)$ profile looks reasonable too. However, it is quite difficult to make conclusions about method's quality based on one example.

Noordermeer et al. (2008) also avoid exponential parametrization and solve a system of equations directly with one additional condition $\sigma_{z}=\sigma_{\varphi}$. The authors also supposed the ratio $\sigma_{z} / \sigma_{R}$ to be constant and varied it in a range from 0 to 0.82 . Noordermeer et al. (2008) used this ratio for reconstructing the gas rotation curve from the asymmetric drift equation. Despite the good result, authors noted that their assumption had no real physical basis.

We revise the procedure of extracting the SVE from the line-of-sight kinematics, consistently rejecting some assumptions made in previous works.

The outline of this paper is as follows.

In Section 2, there is a list of four spiral galaxies with spectral data for the major and minor axes of a disc. In Section 3, a general scheme for SVE reconstruction is given. In Section 4, we describe how the procedure of SVE extracting was modified. In Section 5, some tests and main results are presented. Section 6 discusses the results and related questions, and Section 7 contains conclusions.

\section{DATA}

Our dataset consists of three lenticular S0 (NGC 1167, NGC 3245 and NGC 4150) and one Sab galaxies - NGC 338 (see Table 1). The data for NGC 1167 and NGC 4150 have been provided by Alexei Moiseev from the Special Astrophysical Observatory of the Russian Academy of Sciences and the data for NGC 338 and NGC 3245 have been received from Ivan Katkov (Sternberg Astronomical Institute of the Moscow State University. Other three galaxies from these papers were not included because we select only galaxies with well-defined data across minor axis. The data were obtained with the SCORPIO (Afanasiev \& Moiseev 2005) focal reducer in the long-slit mode (with the slit size of $6^{\prime} \times 1^{\prime \prime}$ ) mounted at the primary focus of the 6-m telescope of the Special Astrophysical Observatory. Observations and data reduction are described in detail in Zasov et al. (2008) for NGC 1167 and NGC 4150 and in Zasov et al. (2012) for NGC 338 and NGC 3245.

Observations were made in the wavelength range 4800-5540 $\AA$, which contains numerous absorption lines of the old stellar populations in the galaxies. The spectral resolution was $2.2 \AA$ for NGC 1167 and NGC 4150 and $2.6 \AA$ for NGC 338 and NGC 3245.

The classical cross-correlation method, similar to employed in Moiseev (2001) was used to calculate the radial velocities and the stellar velocity dispersions for NGC 1167 and NGC 4150. The ULySS software package was used for the pixel by pixel approximation of the observed spectra by the model spectra of stellar populations for NGC 338 and NGC 3245. For this purpose, the high-resolution PEGASE.HR (Le Borgne et al. 2004) models with SSP (Simple Stellar Population) were applied to binarized spectra for all galaxies. Adaptive binning of the spectra along the slit was used with the signal-to-noise ratio in each element not less than 20-50. At every spectra bin fitting produces radial velocity $V_{r}$ and the velocity dispersion $\sigma$. 
Below we listed all the galaxies with their peculiarities. This is a summary of the analysis done by Zasov et al. (2008, 2012).

NGC 338 is a type $\mathrm{Sa}$ or Sab galaxy. The images from Sloan Digital Sky Survey show blurred spiral structures in the disc. The bulge is anomalously bright and extended according to Noordermeer \& van der Hulst (2007) but this conclusion may be due to the inaccurate photometric decomposition of brightness. The galaxy contains a large (for lenticular galaxies) amount of HI gas.

NGC 338 is a fast rotating galaxy with maximum circular velocity near $280-320 \mathrm{~km} / \mathrm{s}$. The velocity profiles were obtained from absorption lines and emissions in $\mathrm{H}_{\beta}$, [NI] and [OIII]. Both gas and stellar rotation curves are measured up to $50^{\prime \prime}$ and have velocity uncertainties not greater than $10 \mathrm{~km} / \mathrm{s}$. The rotation curve reaches the plateau at $r \approx 4 \mathrm{kpc}\left(12.5^{\prime \prime}\right)$, but velocities at distances from the centre larger than $4 \mathrm{kpc}$ become asymmetrical. Such an asymmetry in the velocity field may be due to a small merging event. Gas velocities exceed stellar ones noticeably. This fact can be explained by a large velocity dispersion. The inclination and the position angle are poorly constrained from kinematics and were derived from the optical isophotal analysis (Noordermeer et al. 2007). The line-of-sight velocity dispersion value along the major axis $\left(\mathrm{PA}=108^{\circ}\right)$ is high inside the bulge-dominated region $(r<$ $\left.15^{\prime \prime}\right)$ and then decreases rapidly until it becomes constant at $20^{\prime \prime}$. The velocity dispersion along the minor axis $\left(\mathrm{PA}=17^{\circ}\right)$ has uncertainties about $15-20 \mathrm{~km} / \mathrm{s}$.

NGC 1167 does not belong to any group and does not have satellites with comparable luminosity. The bulge has a steep photometric profile (the Sérsic index $n \approx 3$ according to Zasov et al. 2008) and dominates over the disc up to $15-20^{\prime \prime}$. The total disc luminosity is three times greater than the bulge luminosity. According to Noordermeer et al. (2005), the galaxy contains a significant amount of $\mathrm{HI}$ (the total mass $\approx 1.7 \times 10^{10} M_{\odot}$ ) that is spread over a large area. The average HI surface density remains below the critical value for the gravitational instability of gaseous layer.

Observations of neutral hydrogen show a rotation curve extended over the 10 exponential disc scales. The maximum circular velocity is very large and is not far from $400 \mathrm{~km} / \mathrm{s}$ (at $\left.100^{\prime \prime}\right)$. Stellar rotation velocities are measured up to $r=50^{\prime \prime}$ and are similar to gas values and uncertainties (10-15 km/s). Line-of-sight velocity dispersion profiles along the major and minor axes have average uncertainties around $20 \mathrm{~km} / \mathrm{s}$. They also have the same slope and their central values are close. All these factors do not allow us to separate the profiles from each other in central parts with sufficient confidence.

The inclination and the position angle for this galaxy were derived from the analysis of the velocity field (Noordermeer et al. 2007).

NGC 3245 is an isolated galaxy with the inner and outer discs. The border between the bright inner and outer discs is very sharp and falls on $r \approx 15-20^{\prime \prime}$. No spirals, HII areas and active star formation regions were found. Galaxy has a compact bulge with effective radius approximately equals $5^{\prime \prime}$.

The rotation curve and the velocity dispersion profiles are symmetrical, but the galaxy has some dynamical peculiarities. First, it may be possible that the gaseous disc is inclined to the stellar disc, because gas velocities along the minor axis have non-zero gradient. Second, the stellar rotation curve has a maximum at $r \approx 2.5 \mathrm{kpc}$ (around 30") and slowly decreases after. Also galaxy has huge central velocity dispersion values (similar to NGC 1167, see Figure 1). The line-of-sight velocity dispersion profiles have small uncertainties but they diverge in the centre $\left(\left|\sigma_{\text {los }}^{\text {maj }}-\sigma_{\text {los }}^{\min }\right| \approx 20 \mathrm{~km} / \mathrm{s}\right)$. Perhaps this indicates that the slits were shifted relative to the centre.

NGC 4150 is a low-luminosity galaxy and has the exponential photometric profile. There is no data obtained for the gas motions. The stellar rotation curve remains nearly flat for $r>20^{\prime \prime}$ and has maximum velocity $\approx 100 \mathrm{~km} / \mathrm{s}$. The velocity dispersion data are very noisy and have the biggest uncertainties in the whole dataset. Due to these reasons, the fitting of the line-of-sight velocity dispersion profiles becomes complicated. For NGC 3245 and NGC 4150 the photometrically determined orientation angles of the disc were used.

\section{STELLAR VELOCITY DISPERSIONS}

In cylindrical polar coordinates $(R, \varphi, z)$ the line-of-sight velocity dispersion along a slit relates to the SVE components of a thin disc $\sigma_{R}, \sigma_{\varphi}, \sigma_{z}$ as

$\sigma_{\text {los }, \phi}^{2}=\left[\sigma_{R}^{2} \sin ^{2} \phi+\sigma_{\varphi}^{2} \cos ^{2} \phi\right] \sin ^{2} i+\sigma_{z}^{2} \cos ^{2} i$,

where $\phi$ is the angle between the slit and the major axis of the projected stellar disc (the position angle), $i$ is the galaxy inclination angle. For the observed line-of-sight velocity dispersions along minor and major axes we can write:

$$
\begin{aligned}
\sigma_{\text {los,min }}^{2} & =\sigma_{R}^{2} \sin ^{2} i+\sigma_{z}^{2} \cos ^{2} i, \\
\sigma_{\text {los, } \text { maj }}^{2} & =\sigma_{\varphi}^{2} \sin ^{2} i+\sigma_{z}^{2} \cos ^{2} i .
\end{aligned}
$$

Equations (1) and (2) are valid for a thin disc. For discs at high inclination the assumption about a thin disc in these equations may breaks down as soon as the line-of-sight integration through the disc involves $\sigma_{R}$ from substantial regions through the disc but not from a given $R$. That is why the usage of the above equations for discs at high inclinations seems to be unreliable.

The system of two equations contains three unknowns $\sigma_{R}$, $\sigma_{\varphi}$ and $\sigma_{z}$. It may be closed by some additional dynamical relation, which is valid if the whole system is in equilibrium. One such relation connects the radial and the azimuthal velocity dispersion components with the mean azimuthal velocity of stars $\bar{v}_{\varphi}$ (Binney \& Tremaine 2008)

$f(R)=\frac{\sigma_{\varphi}^{2}(R)}{\sigma_{R}^{2}(R)}=\frac{1}{2}\left(1+\frac{\partial \ln \bar{v}_{\varphi}}{\partial \ln R}\right)$.

Due to the noise presented in the data it is difficult to obtain all three components of the velocity dispersion directly from three equations presented above (Gerssen et al. 1997) because the procedure includes subtraction of very close values $\sigma_{\text {los,min }}^{2}$ and $\sigma_{\text {los,maj }}^{2}$. A possible solution is to parametrize the profiles and to find the best-fitting parameters. Such an approach has been used in many papers (Gerssen et al. 1997, 2000; Shapiro et al. 2003; Gerssen \& Shapiro Griffin 2012), but it depends on the selected parametrization, which is usually exponential. Noordermeer et al. (2008) introduced less parametric approach using equation for asymmetric drift (hereafter AD) and non physical assumption that $\sigma_{\varphi}=\sigma_{z}$. Equation for AD shows difference between the mean stellar rotation velocity and the local circular speed (Binney \& Tremaine 2008):

$v_{\mathrm{c}}^{2}-\bar{v}_{\varphi}^{2}=\sigma_{R}^{2}\left(\frac{\sigma_{\varphi}^{2}}{\sigma_{R}^{2}}-1-\frac{\partial \ln \Sigma_{\mathrm{s}}}{\partial \ln R}-\frac{\partial \ln \sigma_{R}^{2}}{\partial \ln R}\right)$,

where $\Sigma_{\mathrm{s}}$ is the stellar surface density. Notice that this form of the equation neglects the tilt term $d\left(\overline{v_{R} v_{z}}\right) / d z$, which is assumed to be small compared to the other terms. The method by 

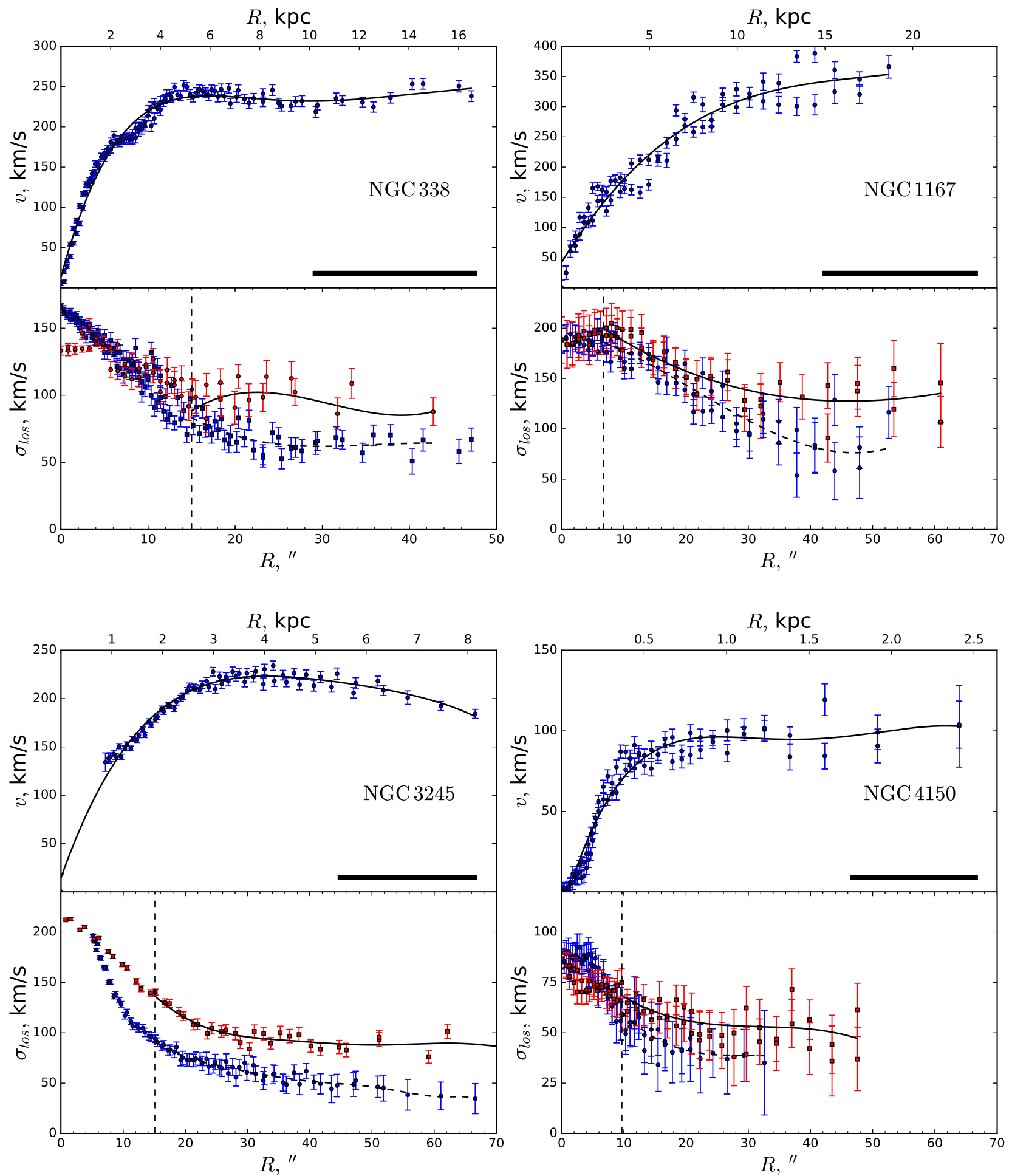

Figure 1. The radial velocities and the velocity dispersions for the examined galaxies. Each galaxy is represented by two plots. The top plot shows the observational points for the stellar rotation curve, their approximation by splines is shown by the solid line. In the lower right corner a line segment is plotted. Its length corresponds to the disc scalelength (see Table 1). The bottom plot shows the observational data for the line-of-sight stellar velocity dispersions along the major axis $\sigma_{\text {los,maj }}$ (filled blue circles) and along the minor axis $\sigma_{\text {los,min }}$ (filled red squares). The solid and dashed lines are the best approximation of the profiles along the minor and major axes, respectively. The vertical dashed line separates the region $R<r_{\mathrm{e}, \mathrm{b}}$ where bulge dominates. 
Table 1. The main parameters of the examined galaxies.

\begin{tabular}{cccccccc}
\hline Galaxy & Type & Inclination $i$, degr & Distance, Mpc & scale, kpc/arcsec & $M_{\mathrm{B}}$ & $r_{\mathrm{e}, \mathrm{b}}, \operatorname{arcsec}$ & $h, \operatorname{arcsec}$ \\
\hline NGC 338 & Sab & $64^{\mathrm{I}}$ & 62 & 0.292 & -21.48 & $15.0^{\mathrm{a}}$ & $18.3^{\mathrm{a}}$ \\
NGC 1167 & S0 & $36^{\mathrm{I}}$ & 66 & 0.310 & -21.73 & $6.7^{\mathrm{a}}$ & $24.2^{\mathrm{a}}$ \\
NGC 3245 & S0 & $62^{\mathrm{II}}$ & 22.3 & 0.107 & -20.17 & $15.1^{\mathrm{b}}$ & $21.6^{\mathrm{b}}$ \\
NGC 4150 & S0 & $56^{\mathrm{II}}$ & 6.7 & 0.033 & -18.51 & $9.5^{\mathrm{c}}$ & $19.7^{\mathrm{c}}$ \\
\hline
\end{tabular}

Morphological type (2) was taken from Zasov et al. (2008) and Zasov et al. (2012). Inclination angle (3) references are (I) Noordermeer et al. (2007), (II) LEDA database (Lyon/MeudonExtragalactic Database). The distance to the galaxy (4) and the scale (5) were found in the NASA / IPAC Extragalactic Database (NED). Data for the Hubble constant $H_{\mathrm{O}}=73.00 \mathrm{~km} / \mathrm{s} / \mathrm{Mpc}, \Omega_{\text {matter }}=0.27, \Omega_{\text {vacuum }}=0.73$. The absolute magnitude in the $B$ band (6) was taken from the LEDA database. Photometric decomposition data, namely the effective bulge radius (7) and the exponential disc scale (8), were taken from (a) Noordermeer \& van der Hulst (2007) in the $R$ band, (b) Fisher \& Drory (2010) and (c) Fabricius et al. (2012) in $3.6 \mu \mathrm{m}$.

Noordermeer et al. (2008) was adopted by Sil'Chenko et al. (2011) for the exponential velocity dispersion profiles and applied to NGC 7217 and its spectral data. Sil'Chenko et al. (2011) supposed to use the brightness for the old stellar population in the $I$ band instead of the surface density in equation (4) and then found the $\sigma_{R}$ profile iteratively.

In this paper we present a new approximation of the line-of-sight velocity dispersion profiles without exponential fits. We adopt only one additional condition that the ratio $\sigma_{z} / \sigma_{R}$ is constant or piecewise constant throughout the disc. This statement is questionable as it is an oversimplification.

Using this assumption, we infer from the first equation in equations (2) that $\sigma_{\text {los,min }}$ is proportional to $\sigma_{R}$ with a proportionality factor $\sin ^{2} i+\alpha^{2} \cos ^{2} i$, where $\alpha$ hereafter means the ratio of vertical and radial velocity dispersions $\sigma_{z} / \sigma_{R}$. This leads to conclusion that for every radius $R$ the next equality is true:

$F(R)=\frac{\sigma_{\mathrm{los}, \min }(R)}{\sigma_{\mathrm{los}, \min }\left(R_{0}\right)}=\frac{\sigma_{R}(R)}{\sigma_{R}\left(R_{0}\right)}$,

for any $R_{0}$ in a suitable range. The selection of the $R_{0}$ value is described in the next section. We define $\sigma_{R, 0}=\sigma_{R}\left(R_{0}\right)$. From the proportion above and from (2) we can derive the final set of equations with two unknown variables $\alpha$ and $\sigma_{R, 0}$ :

$$
\begin{aligned}
& \sigma_{\text {los, } \min }^{2}=\sigma_{R, 0}^{2} F^{2}\left(\sin ^{2} i+\alpha^{2} \cos ^{2} i\right), \\
& \sigma_{\text {los }, \text { maj }}^{2}=\sigma_{R, 0}^{2} F^{2}\left(f \sin ^{2} i+\alpha^{2} \cos ^{2} i\right) .
\end{aligned}
$$

In the next sections we use equations (3) and (6) to derive $\operatorname{SVE}\left(\sigma_{R}\right.$, $\sigma_{z}, \sigma_{\varphi}$ ) and invoke the $\mathrm{AD}$ equation (4) to verify radial dispersion values when it is possible.

\section{THE METHOD}

The stellar rotation curves were corrected for the mean velocity, folded around the centre and reduced for the inclination factor $\sin i$. The resulting curves were fitted by the best smoothing B-spline curve with $k=3$ which is stay unchanged in further analysis. The same corrections and approximation have been applied to the gas data if they were available.

Both stellar velocity dispersion profiles along the major and minor axes were bended around the appropriate centre. After that $\sigma_{\text {los }}^{\min }$ data were deprojected and reduced for the inclination factor $\cos ^{-1} i$. Then, all data at radii less than effective bulge radius $r_{\mathrm{e}, \mathrm{b}}$ were removed to avoid the bulge influence in the central region and contamination of profiles. Photometric scalelengths for the examined galaxies are listed in Table 1. Both line-of-sight velocity dispersion profiles were fitted by smoothing B-spline with $k=3$ in the range from $R_{0}$ to the last point of the data. In order to take into account different errors in each approximation we use shifted inverse square weight function defined as $w^{-1}(R)=1+\delta \sigma^{2}$, where $\delta \sigma$ is the data inaccuracy for the velocity dispersion at radius $R$. In Fig. 1 the solid line presents the approximation of the data for the minor axis, and dashed line refers to the data for the major axis. The dashed line is drawn to show the difference between two profiles, as we do not use the fitting of the major axis data in our analysis.

The ratio $\sigma_{\varphi}^{2} / \sigma_{R}^{2}$ in equation (3) was calculated directly using derivative for the spline approximation of $\bar{v}_{\varphi}$. Since we use a smooth spline approximation for the rotation curve the derived profile $f(R)=\sigma_{\varphi}^{2}(R) / \sigma_{R}^{2}(R)$ also stays smoothed for all galaxies. Note that values of $f(R)$ usually lie between 1 and 0.5 , because in the central parts, where the disc rotates solidly, $f(R)=1$ from equation (3) and at the periphery, where $\bar{v}_{\varphi}$ is almost constant, $f(R)$ is close to 0.5 .

If the approximations for the profiles $\bar{v}_{\varphi}$ and $\sigma_{\text {los,min }}$ are fixed $(F(R)$ is also fixed), the system of equations (6) becomes linear with two unknowns $\sigma_{R, 0}$ and $\alpha$. The unknown $\sigma_{R, 0}$ depends on the choice of the point $R_{0}$. We can choose any $R_{0}$ within an appropriate range. If we take the point $R_{0}^{\prime}$ instead of $R_{0}$, it will be equivalent to the linear substitution of the unknown in the original system of equations (6) because of the equality (5). Thus, the solution of the system does not depend on the choice of $R_{0}$. Here, and after, we assume the value of the radial velocity dispersion at the effective bulge radius i.e. $\sigma_{R, 0}=\sigma_{R}\left(r_{\mathrm{e}, \mathrm{b}}\right)$ as an unknown, unless otherwise specified.

Because of noisy data profiles, trying to solve the system of equations (6) directly is unreasonable and may led to ambiguous results. That is why we scanned the space of all possible values of unknowns and plot the maps of reduced $\chi^{2}$ values, i.e. weighted sum of squared errors divided by the number of degrees of freedom. We varied $\sigma_{R, 0}$ and $\alpha$ in the appropriate range and for every pair of $\sigma_{R, 0}$ and $\alpha$ we calculated $\chi^{2}$ for the data and expected values predicted by equation (6). It was done for both axes data lying after the bulge effective radius $r_{e, b}$. From these maps, we can see directly whether there is evidence for degeneracy or for the optimal parameters existence. We chose the range for parameter $\alpha$ as $0.25 \leq \alpha \leq 1.0$, where the lower boundary is fixed at the level just near that given by the local linear criterion for the bending instability (e.g. Rodionov \& Sotnikova 2013). The upper boundary is generally accepted and has two motivations. On the one hand, there are some agent in a galaxy that could 'transfer' in-plane velocity dispersion into the vertical one making a disc thicker. It could be giant molecular clouds (GMCs) that are usually thought as a 'three-dimensional heating agent'. (Lacey 1984) found that when stars are scattered by GMCs, a ratio $\alpha \approx 0.8$ is rapidly established. On the other hand, in the absence of a third integral of motions, the ratio $\sigma_{z} / \sigma_{R}$ to be equal to 1 (Binney \& Tremaine 
2008). The varying step was equal to 0.01 . For second parameter $\sigma_{R, 0}$ boundaries were zero as a lowest possible value and $500 \mathrm{~km} / \mathrm{s}$ as an upper limit. The varying step was $0.25 \mathrm{~km} / \mathrm{s}$.

Gerssen et al. (1997, 2000); Shapiro et al. (2003); Gerssen \& Shapiro Griffin (2012) used the additional AD equation for including in dispersion's $c h i^{2}$ also the gas rotation curve (fitted by power function). They obtain a best fit for five-parameters model, while we search in two-dimensional space only, using fixed spline approximations for data profiles, including star rotation curve. Thus proposed method modification is less model-specified and can handle profiles with peculiarities, which are not fitted well by exponential or power law (like NGC 338). However, it has not used additional information and constraint from gas data.

For the obtained values of $\sigma_{R, 0}$ and $\alpha$ we can retrieve all components of SVE. The profiles of $\sigma_{\text {los,min }}(R)$ and $\sigma_{\text {los,maj }}(R)$ can be directly obtained from equation (6). The stellar velocity dispersion profile $\sigma_{R}$ is equal to $\sigma_{R, 0} \times F(R)$ by definition. The vertical velocity dispersion profile $\sigma_{z}(R)$ can be obtained as $\sigma_{R}(R)$ multiplied by a factor $\alpha$. Multiplication of $\sigma_{R}(R)$ and $f(R)$ from equation (5) produces the azimuthal dispersion profile $\sigma_{\varphi}(R)$.

\section{RESULTS}

\subsection{NGC 1068}

In order to check whether our method is working and to show the representation of our results for all galaxies, we took galaxy NGC 1068 from a different sample (Shapiro et al. 2003). This sample contains another three galaxies, NGC 2460, NGC 2775 and NGC 4030. While NGC 2775 we use in analysis below, other two galaxies we consider insufficient for it because of small number of observational points and short profile's radial region. Selected for test NGC 1068 is Sb type galaxy with the inclination $30 \pm 9$ degrees. The velocity dispersion profiles and the rotation curve lie in the $38-90^{\prime \prime}$ range and the photometric scale for this galaxy is equal to $21^{\prime \prime}$. Shapiro et al. (2003) suggested the exponential profiles for the radial and vertical velocity dispersions with the same scale length. They found the optimal model that minimizes the total $\chi^{2}$ for both $\sigma_{\text {los }}$ profiles and for the AD profile simultaneously. In Shapiro et al. (2003) the best fit was reached for $\sigma_{z} / \sigma_{R}=0.58 \pm 0.07$ and for $\sigma_{R, 0}(0)=213 \pm 20 \mathrm{~km} / \mathrm{s}$.

Although, we used the spline fitting instead of exponential functions our approximations of the profiles resemble those obtained in Shapiro et al. (2003). The $\chi^{2}$-maps for these approximations applied for the minor and major axes are presented in Fig. 2. The unknown $\sigma_{R, 0}$ is the value of the stellar radial velocity dispersion at $38.5^{\prime \prime}$ radii, which corresponds to the distance from the galaxy centre to the first observational point. To demonstrate the existence of the global minimum, we selected the minimal $\chi^{2}$ value for both unknowns $\left(\sigma_{R, 0}\right.$ and $\left.\alpha\right)$ for the minor axis with a narrow strip around this value. Then we put this strip over the $\sigma_{\text {los,maj }}$ map. In Fig. 2 this strip is filled by grey and contains all $\chi^{2}$ values that are smaller or equal to the minimal $\chi^{2}$ plus $10 \%$. The minimal reduced $\chi^{2}$ value for $\sigma_{\text {los,min }}$ is the solution of the first equation in equations (6). We drew it as a dotted line. Values of $\chi^{2}$ for the major axis were received for each pair of parameters $\sigma_{R, 0}$ and $\alpha$ from the grey area and are shown in the bottom subplot in Fig. 2. The dotted line is not completely inside the grey area. For example, for $\alpha=0.5$, two borders of the grey zone intersect the same isoline while dotted line has just crossed it and now corresponds to a smaller value of $\chi^{2}$ (the middle subplot in Fig. 2).
The maps have illustrative character and the width of the grey strip was chosen arbitrary. We use these maps because they show the errors of fitting for both axes independently. In order to determine the global minimum correctly, the mutual $\chi^{2}$-map for both axes should be used. It is clear that the $\chi^{2}$ minimum for both axes is reached exactly at the same region where lies the minimum for parameters from the grey area in the bottom subplot. We plot mutual $\chi^{2}$-maps for all galaxies in sample except NGC 1167. Such maps are shown in Fig. 3-5.

The constructed maps show that the velocity dispersion profiles are not restored individually, as parameters are degenerate. In other words, for any value of $\alpha$ one can find a suitable value of $\sigma_{R, 0}$ so that an error corresponding to the recovered profile will be minimal. For NGC 1068 as well as for all other galaxies under consideration, the degeneracy along the minor axis is stronger, i.e. the choice of the appropriate values of parameters gives the same $\chi^{2}$ for all $\alpha$. Such a behaviour follows directly from the first equation in equations (6), where the connection between $\alpha$ and $\sigma_{R, 0}$ can be explicitly written in the analytical form. Nevertheless, it is clear from Fig. 2 that a global minimum exists. In other words, there are values for $\alpha$ and $\sigma_{R, 0}$ that minimize errors for both data projections simultaneously. The range for the minimal values for $\alpha$ is wide enough but still lies inside confidential interval for this parameter from Shapiro et al. (2003). The obtained value for the radial velocity dispersion $\sigma_{R, 0}$ is consistent with the value determined by Shapiro et al. (2003) after we sample their radial velocity dispersion at $R_{0}$ (see below).

The result was proven to be stable with Monte-Carlo method using 10000 random realizations of data. For each realization and each point along a slit a new "observable" was chosen randomly from the normal distribution centred at the original value and with the dispersion equal to the half of the original uncertainty. For this new dataset, new approximations were built and new profiles $F(R)$ and $f(R)$ were derived. Then we sorted out all possible parameters and found optimal values of $\alpha$ and $\sigma_{R, 0}$ so that mutual $\chi^{2}$ for both profiles $\sigma_{\text {los,min }}$ and $\sigma_{\text {los,maj }}$ would be minimal. Such optimal parameters correspond to the global minimum in our maps of $\chi^{2}$. The cloud of 10000 optimal parameter pairs was sufficiently compact and provides final values of $\alpha$ and $\sigma_{R, 0}$ with $1 \sigma$ error: $\alpha=0.62 \pm 0.07, \sigma_{R, 0}=134 \pm 9$. The second parameter $\sigma_{R, 0}$ differs from that obtained in Shapiro et al. (2003), because we considered $\sigma_{R, 0}$ at $38.5^{\prime \prime}$ radius. In order to compare the radial velocity dispersion values between different models we should correct them for distance to the centre. For this galaxy the kinematic exponential scale length obtained in Shapiro et al. (2003) is equal to $72^{\prime \prime}$, hence a correction factor is equal to $\exp (-38.5 / 72$.) and produces the final value of $\sigma_{R, 0}$ at $R=38.5^{\prime \prime}$ to be equal to $125 \mathrm{~km} / \mathrm{s}$. This final value is very close to that obtained in our analysis.

\subsection{Examined galaxies}

We obtained similar maps and performed Monte-Carlo checking as before for all galaxies from the Table 1. Obtained results show no evidence of global minimum existence, except one galaxy. All maps for examined galaxies are drawn in Fig. 3-6 in the same manner as for NGC 1068. The exception is NGC 1167 that has the lowest inclination angle among the galaxies in the Table 1 . For this galaxy, our analysis gave the minimum for $\sigma_{z} / \sigma_{R}$ near the lowest boundary for $\alpha=0.3$. However, this minimum was reached not only in a single point. Values of $\chi^{2}$ stay almost constant in the wide range from lowest $\alpha$ (even for $\alpha<0.3$ ) till $\alpha \approx 0.5$, i.e. minimum is 


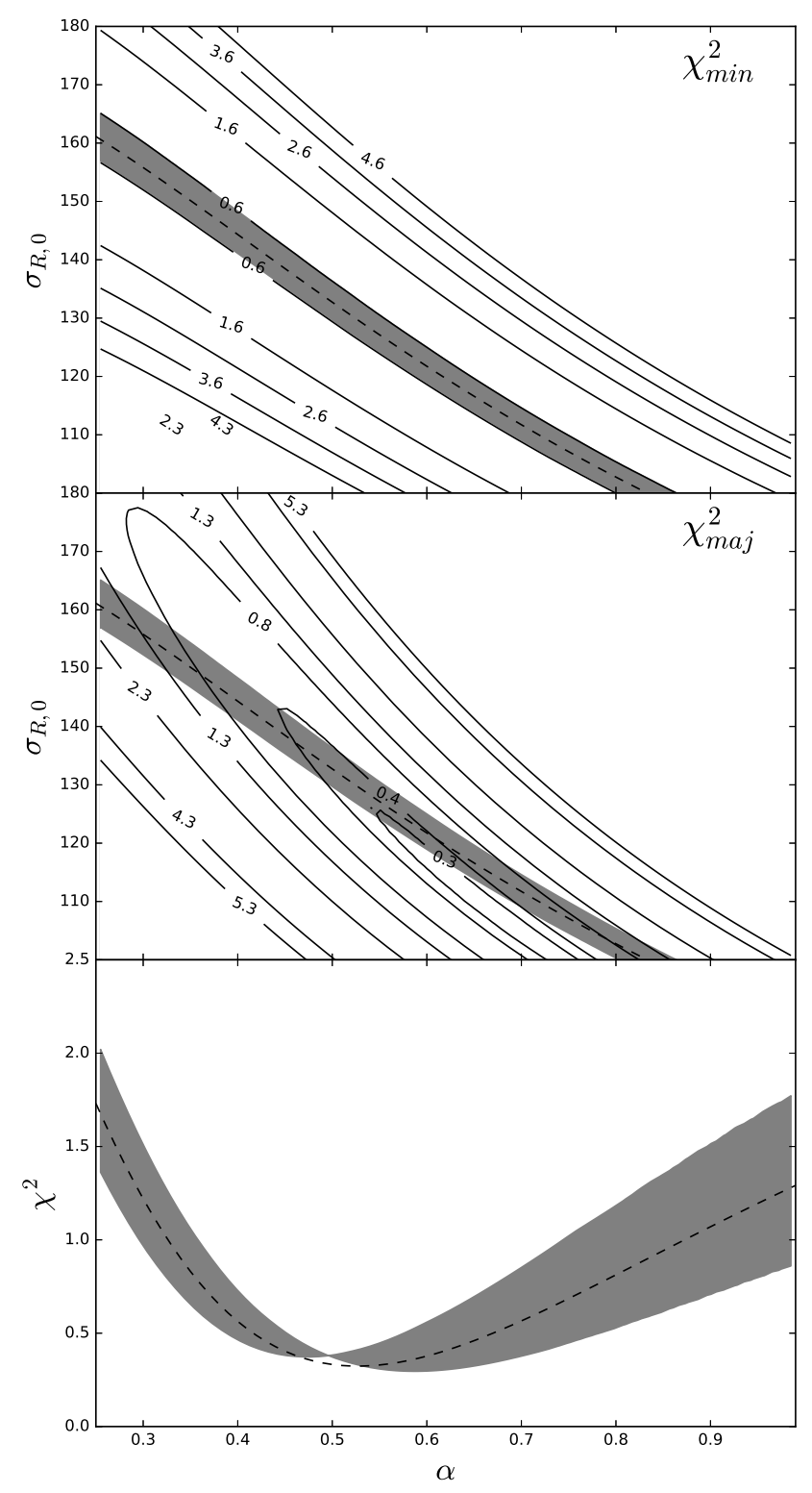

Figure 2. Maps of $\chi^{2}$ error for NGC 1068. In the upper and middle plots $\chi_{\text {min }}^{2}$ and $\chi_{m a j}^{2}$ maps are shown for $\sigma_{\text {los,min }}$ and $\sigma_{\text {los,maj }}$ data respectively. In both maps solid lines represent levels of the same $\chi^{2}$ and the dashed lines show the dependence between $\sigma_{R, 0}$ and $\alpha$ (see text for details). The grey shaded region corresponds to the values of the parameters $\sigma_{R, 0}$ and $\alpha$ which minimize $\chi_{\min }^{2}$ for the line-of-sight stellar velocity dispersion along the minor axis. The bottom plot shows $\chi^{2}$ values, received for $\sigma_{\text {los,maj }}$ profile when parameters are taken from the grey area or along the line relation between $\sigma_{R, 0}$ and $\alpha$ (dashed line).

very vast. Monte-Carlo modeling does not produce any preferable value of $\alpha$ from $\alpha<0.5$ range. Thus, the case of NGC 1167 is not simple and this galaxy will be examined separately in the next section.

For other three galaxies, NGC 338, NGC 3245 and NGC 4150 $\chi^{2}$ monotonically increases with growing $\alpha$. Hence, the lowest $\chi^{2}$ may be found for the lowest $\alpha$ or, in other words, at the left boundary of $\alpha$, as it shown in the bottom plots of the appropriate map. If we consider maps for both axes independently they will demonstrate a degeneracy trend in the same way as for NGC 1068.

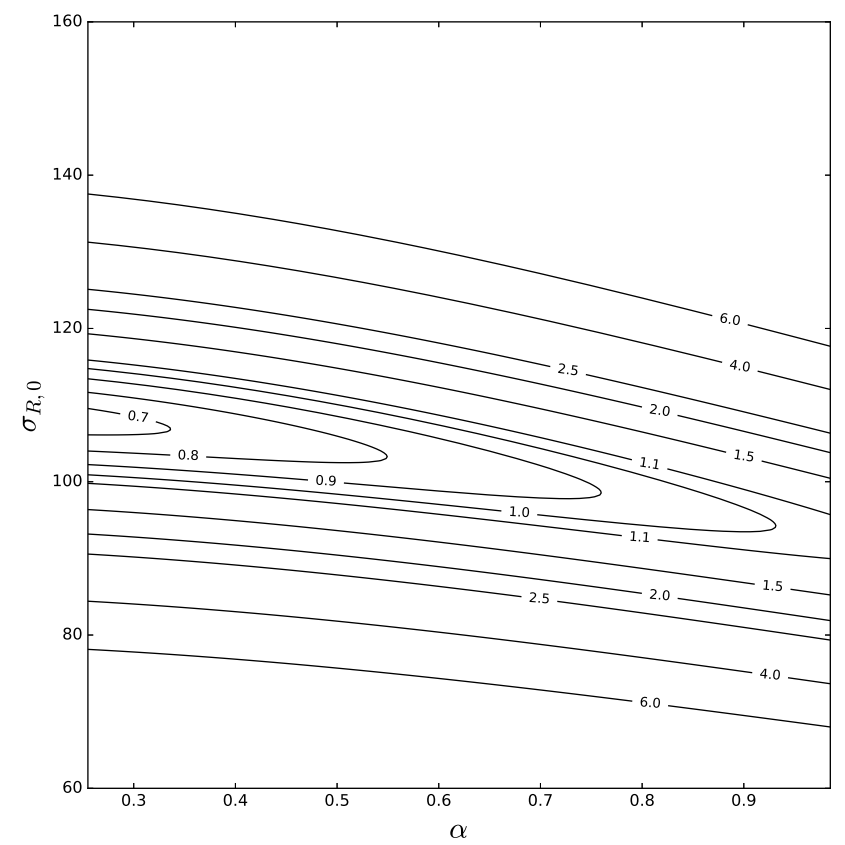

Figure 3. The map of mutual $\chi^{2}$ for the major and minor axes for the galaxy NGC 338. The lines show levels of the same $\chi^{2}$ values with the level value written in the plot near each line.

The difference in $\chi^{2}$ values between these three galaxies may be explained by galaxies' parameters dissimilarity, namely, by different typical uncertainties, different number of data points and so on. For example, NGC 3245 has small relative errors and a large gap between $\sigma_{\text {los,min }}$ and $\sigma_{\text {los,maj }}$ profiles that leading to large $\chi^{2}$ values.

Small optimal values of $\alpha$ are likely not to be naturally occuring and appear as artefacts in numerical models due to the limited range of $\alpha$. These small values were confirmed by Monte Carlo simulations that produce $\alpha$ near to the left border in most realizations. As mentioned in Section 4, $\alpha$ less than 0.3 seems to be unrealistic. Thus, obtained results make questionable our main assumption about small variation of the ratio $\sigma_{z} / \sigma_{R}$. Another possible source of uncertainties is observational data quality. However, presented list of galaxies is not uniform by many parameters and it is unlikely that data quality may cause similar results especially with additional statistical support. Small values of $\alpha$ and difficulties in its extraction from data are due to the small contribution of $\sigma_{z}$ in the observed line-of-sight velocity dispersion profiles when a galaxy has a large inclination. We discuss this in detail in the next section.

\section{DISCUSSION}

The results for three galaxies in the Table 1 differ from the result for NGC 1068. In these galaxies, a strong degeneracy of parameters is observed and all formal optimal values of $\alpha$ are as small as possible in the physically meaningful range. Even for NGC 1167, for which a real global minimum of $\chi^{2}$ may be found ( $\alpha \approx 0.3$ ), more accurate analysis should be done. Received results may relate to the impact of a significant inclination on observational data or may be caused by incorrectness of the main assumption of small changes of $\alpha$ across kinematic profiles. Both possibilities will be examined below. We also discuss whether the boundary values of 


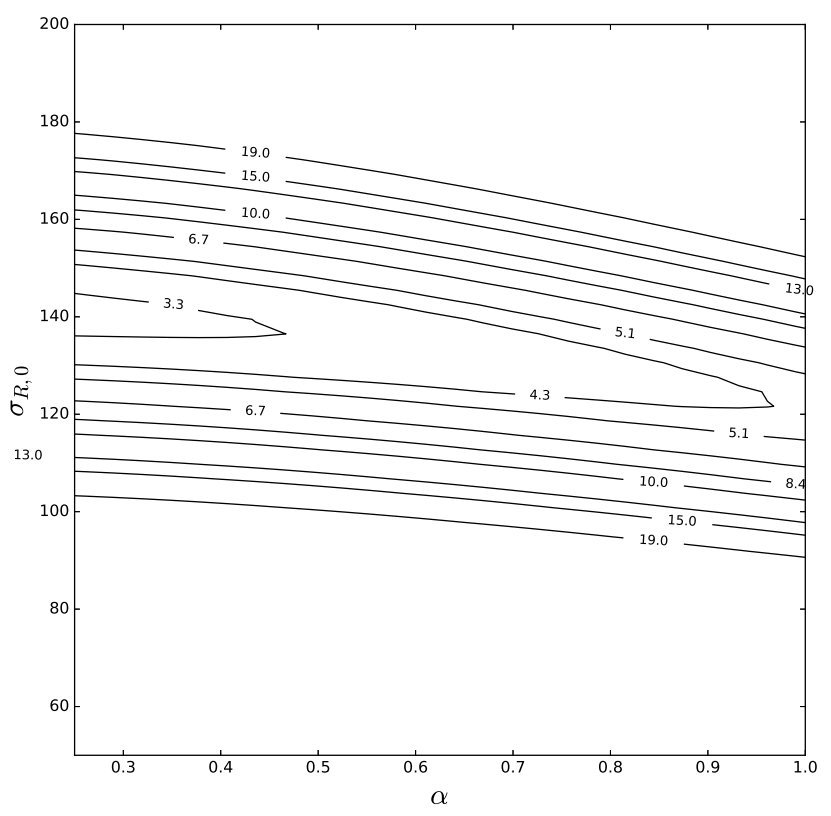

Figure 4. Map of mutual $\chi^{2}$ for NGC 3245 (similar to Fig. 3).

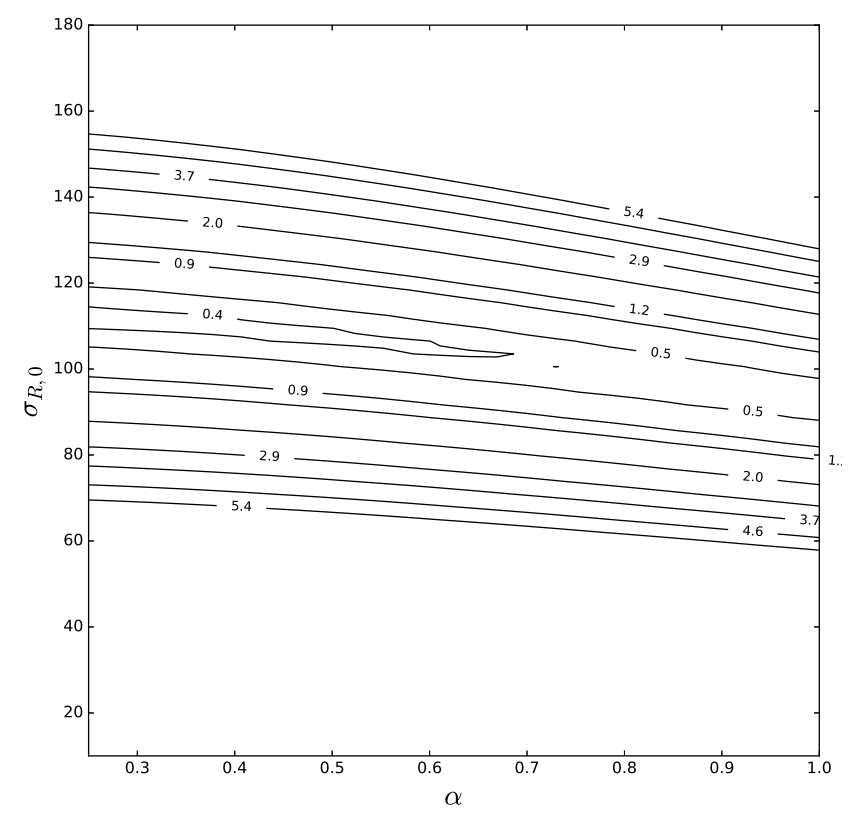

Figure 5. Map of mutual $\chi^{2}$ for NGC 4150 (similar to Fig. 3).

$\alpha$ may be considered as physical. In last part of this section, we estimate the possible range for the radial velocity dispersion values and analyse its consistency with the range constrained by other methods.

The inclination impact may be evaluated from equations (2). According to these equations, the vertical velocity dispersion contributes to the observational data a little when the galaxy inclination is large. Indeed, it is seen from second equation that the stellar line-of-sight velocity dispersion for the minor axis consists of two terms, $\sigma_{R}^{2} \sin ^{2} i$ and $\sigma_{z}^{2} \cos ^{2} i$, that contribute in the resulting sum as $1: \alpha^{2} \operatorname{ctg}^{2} i$. For the inclination angles $i>60^{\circ}$ this leads to the vertical velocity dispersion term contribution not

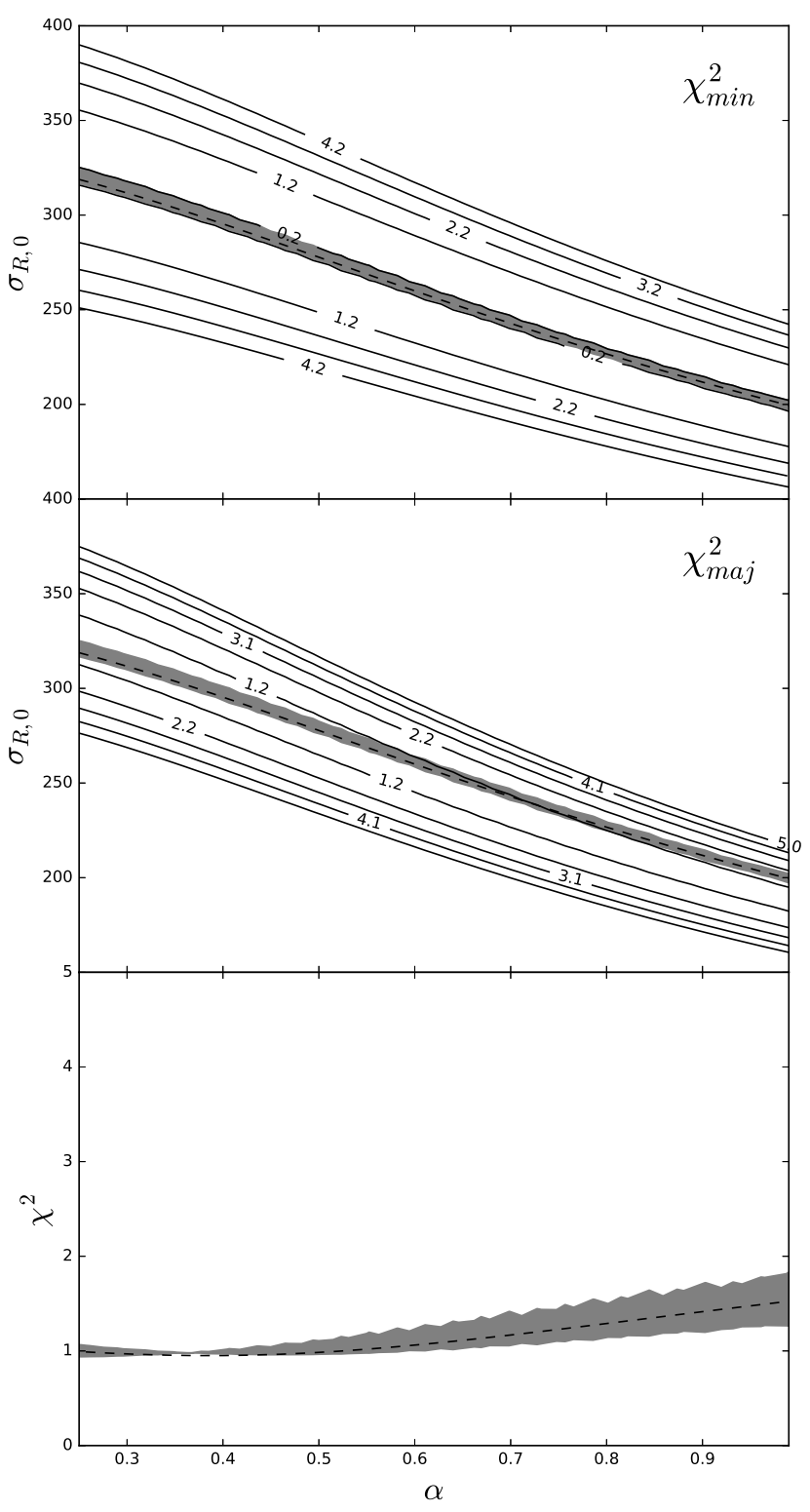

Figure 6. Maps of $\chi^{2}$ for NGC 1167. Notations similar to those in Fig. 2.

greater than one-third. This value should be compared with the observational uncertainties. As shown on Fig. 7, in the case of NGC 338 and NGC 4150 the relative $\sigma_{\text {los,min }}^{2}$ errors are large and thus for every $\alpha \leq 0.7$ the contribution of $\sigma_{z}$ is comparable with observational uncertainties and cannot be correctly extracted. Even for NGC 3245, which has the smallest relative errors in the sample, for $\alpha \leq 0.7$ the vertical velocity dispersion cannot be distinguished from the error for majority of observational points and thus it is also insufficient for the correct reconstruction of SVE. Note that it appears unlikely for our data that it is possible to obtain larger vertical to radial velocity dispersions ratio, because we analyse extended profiles and in the absence of GMCs in the outer part of a galaxy there is only merging that can lead to the high vertical disc heating till distant radii 2-2.5 $\mathrm{h}$. Heating by merger may be true for NGC 338, but NGC 3245 is isolated and NGC 4150 has poor environment.

Thus for galaxies with the inclination $i>60^{\circ}$ and relative data errors similar to ours it is hard or nearly impossible to reconstruct $\alpha$ 


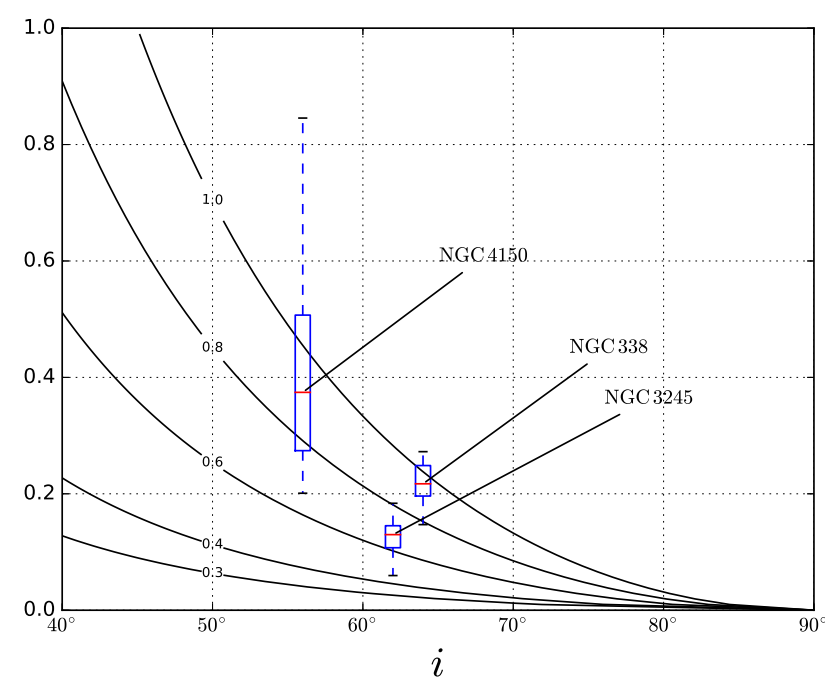

Figure 7. Inclination impact comparison with observational uncertainties. Solid lines show $\alpha^{2} \operatorname{ctg}^{2} i$ for different $\alpha$, which correspond to vertical velocity dispersion term contribution in second equation in equations (2). Distributions of $\sigma_{\text {los min }}^{2}$ relative errors for three annotated galaxies are represented by boxplots, which demonstrate maximum, minimum, median, first and third quartiles of distribution. Every boxplot have the same width and $\mathrm{x}$-coordinate equal to corresponding galaxy's inclination.

values. Second difficulty is that the equation (1) is valid only for a thin disc and does not include the line-of-sight integration of the DF for galaxies with high inclination. Perhaps, this is the reason why we obtain the formal minimal $\chi^{2}$ values for the smallest values of $\alpha$ for NGC 338, NGC 3245 and NGC 4150.

\subsection{The $\alpha$ solution on the edge}

There exists one simple reason why obtained values of $\alpha$ on the edge of the variation range are not realistic. It can be seen from the $\chi^{2}$-maps that for three galaxies NGC 338, NGC 3245 and NGC $4150 \chi^{2}$ continues to decrease outside the left border. If we did not know an additional constraint for the ratio $\sigma_{z} / \sigma_{R}$, we could derive unphysical $\alpha$ values from the analysis. Optimal $\alpha$ values close to their variation boundaries were received in papers by other authors too. For example, the optimal $\alpha$ values were found both equal to the maximal possible value $\alpha=1$ (Shapiro et al. 2003, NGC 2775) and near the left boundary $\alpha=0.25$ for NGC 2280 and $\alpha=0.29$ for NGC 3810 (Gerssen \& Shapiro Griffin 2012). For these three galaxies only NGC 2775 has data long enough for analysis. We analyse it below in order to determine whether such optimal values on the edge were realistic or not.

NGC 2775 is a galaxy of Sa or Sab morphological type and has the inclination angle around $40^{\circ}$. The velocity dispersion profiles in Shapiro et al. (2003) are extended in the wide radial region 31 $62^{\prime \prime}$. They mention that there are differences in bulge contribution evaluations between papers and it is possible that the disc does not dominate in the examined range. However, they believe that the bulge influence becomes negligible for distances greater than $30-$ $40^{\prime \prime}$ from the galaxy centre. The best model fit for $\alpha$ was $1.02 \pm 0.11$.

Our best approximation of the profile $\sigma_{\mathrm{los}, \min }$ is close to the best fit in Shapiro et al. (2003). It may be explained by the fact that observational points lie not far from exponential fit and splines follow the same trend. Thus, it is expected that when we try to modify the method by using exponential profile parametrization

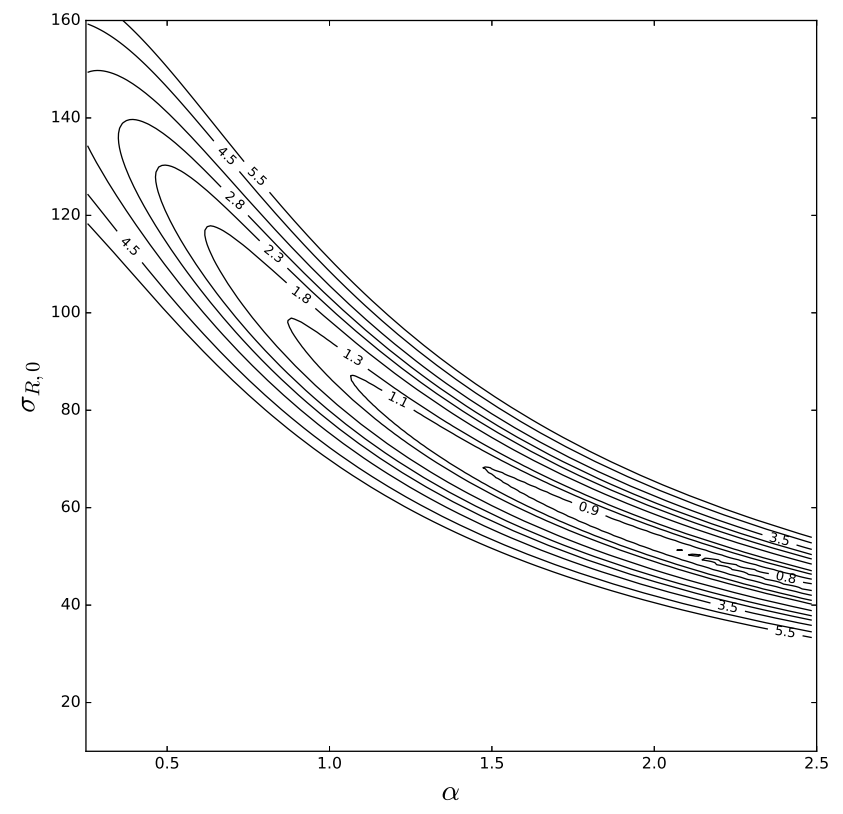

Figure 8. Map of $\chi^{2}$ for NGC 2275 (similar to Fig. 3).

and apply it to NGC 2775, we obtain quite the same results. The rotation curve changed a little at radii under discussion and also was fitted quite similar to the approximation in the original paper. The $\chi^{2}$-maps are shown in Fig. 8. In order to be confident that $\alpha$ being close to the right boundary is not a true minimum, we extended the range of $\alpha$ and plotted the maps for greater $\alpha$ up to $\alpha=2.5$. Fig. 8 shows that minimal $\chi^{2}$ formally reaches for $\alpha=1$ and $\sigma_{R, 0} \approx 90 \mathrm{~km} / \mathrm{s}$, which are close to the values from Shapiro et al. (2003). However, $\alpha=1$ is not a global minimum, since $\chi^{2}$ continues to decrease drastically and reaches notably lower values for larger $\alpha$. We check the stability of this result with 10000 Monte Carlo realizations of the data. For all realizations we found the minimal $\chi^{2}$ value for $\alpha$ to be close to 2.5.

Hence, the galaxy NGC 2775 demonstrates that the optimal $\alpha$ being close to the limit of possible physical values is not a feature of observational data or selected approximations. Despite that we derived the same optimal parameter for $\alpha \leq 1$ (as in Shapiro et al. 2003), we showed that the minimal $\chi^{2}$ value lies far from constrained boundary. In other words, the solution $\alpha=1$ is not real and seems rather to be a numerical peculiarity of the model conditions. We also tried to perform the analysis for NGC 2280 from Gerssen \& Shapiro Griffin (2012) that has small $\alpha$, but failed to reproduce the result as we obtained a high value of $\alpha$.

\subsection{Non-constant $\alpha$ model for NGC 1167}

The galaxy NGC 1167 is inclined at $30^{\circ}$ and thus contribution of $\sigma_{z}$ in $\sigma_{\text {los,min }}$ become significant. In addition, the galaxy demonstrates a real global minimum for $\alpha$ inside the possible range. However, the optimal value $\alpha \approx 0.3$ lies near the boundary where disc stays stable against bending instability (see Section 4). Since there are many dynamical mechanisms for the disc heating in the vertical direction, this minimal value seems to be questionable. The only substantial assumption in our model, which may affect the position of global minimum, is $\alpha=$ const throughout the disc. This may be wrong for extended profiles. Therefore we tried to derive $\alpha$ for two smaller separate pieces of the profiles and compared them. We 
make such an analysis for NGC 1167 and discuss derived results below.

The profile $\sigma_{\text {los,min }}$ demonstrates a bend at about $30^{\prime \prime}$ (Fig. 1). We took this point to split up the profile into two parts and found the best values of $\alpha$ before and after the bend independently. We examined the profile at the ranges from the bulge effective radius $r_{\mathrm{e}, \mathrm{b}}$ till $30^{\prime \prime}$ and from $30^{\prime \prime}$ till the end of the observational data. These pieces of the profile have roughly equal lengths. We approximated them by exponential law because they are well described by such parametrization. Then for every part the $\chi^{2}$-maps were constructed and the optimal values of $\alpha$ were found at the same manner as before. Obtained values of $\alpha$ were tested using the Monte Carlo method with 10000 realizations of the data for every part.

The results of simulations are shown in Fig. 9. It is seen that the clouds of Monte Carlo realizations for different parts are separated clearly from each other. These clouds are relatively compact and well described by a two-dimensional normal distribution for $\sigma_{R, 0}$ and $\alpha$. For the inner part we found $\alpha=0.72 \pm$ 0.09 with $1 \sigma$ error. For the outer part $\left(30-60^{\prime \prime}\right) \alpha=0.30 \pm 0.08$. Second value of $\alpha$ lies close to the limit of the physical parameter range but is probably real. In isolated galaxies, in the absence of a separate agent causing out-plane scattering (GMCs, nearby external galaxies), the initial bending instability, which develops and decays within the first Gyr of the disc evolution, heats outer parts of the disc up to the level corresponding to the linear criterion of the bending instability $\alpha \approx 0.30$ (Rodionov \& Sotnikova 2013).

The decrease in $\alpha$ with rising distance from the centre is consistent with the results of the $N$-body simulations by Zasov et al. (2008). It is also remarkable that our value of $\alpha$ in the inner part is found to be equal to the one obtained in Zasov et al. (2008) using $N$-body simulations (see fig. 8 in the cited paper). For both parts we reconstructed the original line-of-sight velocity dispersion profiles in the manner specified above. These profiles with the acceptable deviations are shown in Fig. 10. Both profiles are consistent with observational data. This fact gives a strong support for the obtained results. Notice that the solid line, which shows the inner reconstructed profile for $\sigma_{\text {los,min }}$ (the bottom plot in Fig. 10), is almost identical to exponential approximations used in the analysis.

It can be noted that optimal $\alpha$ determined for the whole profile is not equal to the average between values of optimal $\alpha$ obtained for two separated parts of the profile and is close to the lowest one. There are two reasons for such behaviour. First, the global minimum for $\alpha \approx 0.3$ is very vast and the change of $\chi^{2}$ is almost not noticeable up to the value of $\alpha \approx 0.5$, which is close enough to the average value. The second reason is that the observed profiles of $\sigma_{\text {los,min }}$ and $\sigma_{\text {los,maj }}$ are significantly diverge at distant regions. If we constrain $\alpha$ between $0.3-0.5$ this results in small $\chi^{2}$ values. Increasing values of $\alpha$ will produce the profile $\sigma_{\text {los,maj }}$ with more and more deviation from the observational data at large distances from the centre that results in the $\chi^{2}$ increase. At the same time, the central part of the major axis profile is approximated rather well for small values of $\alpha$, only slightly increasing the total error. These two reasons explain why $\alpha \approx 0.3$ for the whole profile.

Thus, for NGC 1167, the initial assumption of a constant value of $\alpha$ along the whole profile is less physically motivated. If we reject this assumption we can reconstruct SVE over the entire radial region and find using Monte Carlo simulations the obtained inner and outer values of $\alpha$ and $\sigma_{R, 0}$ to be stable. However, for other galaxies, we did not perform such analysis because for them inclination impact cannot be omitted even for splitted profile.

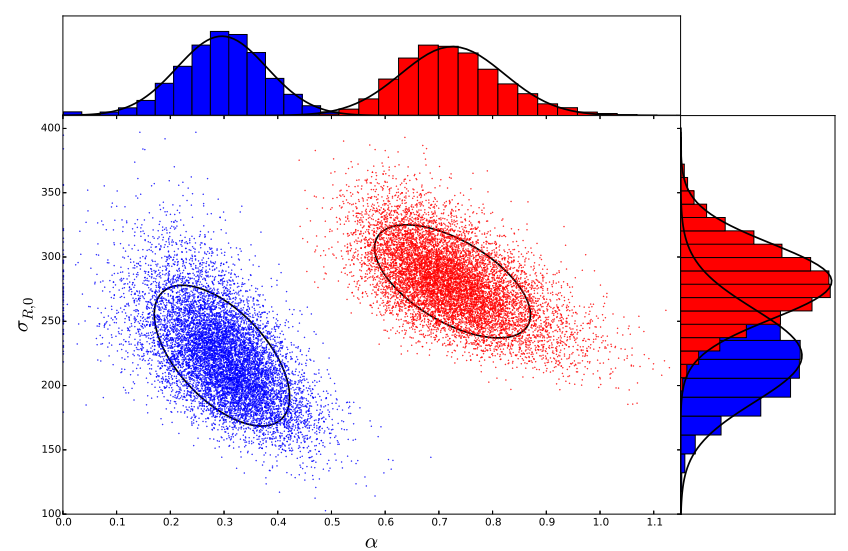

Figure 9. The results of Monte-Carlo simulation for galaxy NGC 1167 for the profile $\sigma_{\text {los,min }}$ divided in two parts. Each point shows optimal values of $\alpha$ and $\sigma_{R, 0}$ for a particular realization of data that minimizes mutual $\chi^{2}$. A cloud with red dots and greater $\alpha$ corresponds to the inner part of the profile while the blue dots with smaller $\alpha$ correspond to the outer part. A solid ellipse for each cloud demonstrates the level of one standard deviation from the centre of the two-dimensional normal distribution. The upper and right subplots show histograms of each parameter with the solid line that follows the best Gaussian approximation.

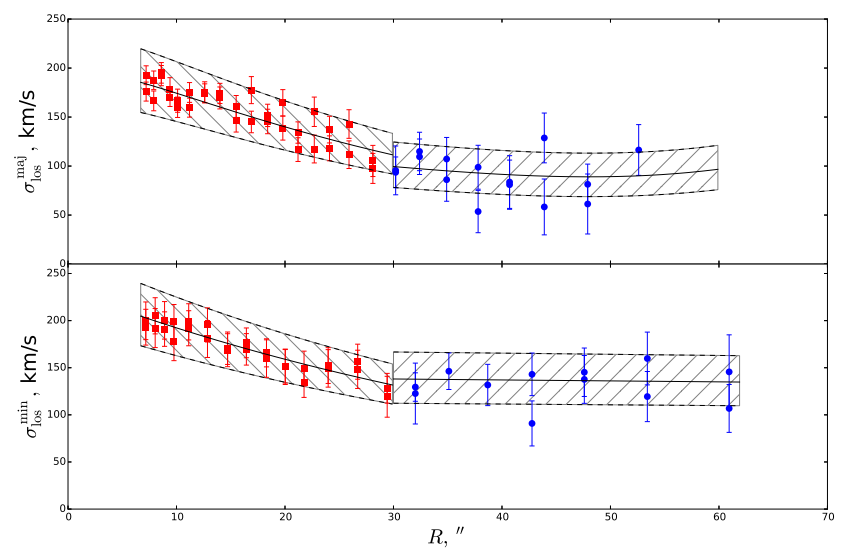

Figure 10. Observed and reconstructed profiles of the line-of-sight velocity dispersions for NGC 1167 in the case of divided profile. The upper plot shows the profile of the line-of-sight velocity dispersions along the major axis $\sigma_{\text {los,maj }}(R)$ and the bottom plot shows the profile along the minor axis $\sigma_{\text {los,min }}(R)$. For both plots, filled symbols indicate observational data (red squares for the inner part and blue circles for the outer one). The solid line represents a reconstructed velocity dispersion profile with parameters equal to the mean values of bivariate normal distributions obtained by Monte Carlo method (see Fig. 9). Shaded area corresponds to all possible model profiles $\sigma_{\text {los,maj }}$ and $\sigma_{\text {los,min }}$ reconstructed for parameters $\alpha$ and $\sigma_{R, 0}$ which differ from its mean values no more than one standard deviation.

\subsection{AD and constraints on $\sigma_{R, 0}$ for NGC 338}

Although we failed to determine the SVE and find the optimal $\alpha$ for the highly inclined galaxies, we can get a constraint to the second parameter $\sigma_{R, 0}$. This constraint follows from the $\chi^{2}$-maps (Fig. 3-5). It is seen, that the factor $\sigma_{R, 0}$ for galaxies with large inclinations changes a little in a wide range of $\alpha$ values. Such a behaviour infers from the first equation in equations (6) where the relation between $\sigma_{R}$ and $\sigma_{\text {los,min }}$ is defined by the value in brackets, depending on two parameters $\alpha$ and $i$. The ratio $\alpha=\sigma_{z} / \sigma_{R}$ can formally vary between 0 and 1 . Then, the value in brackets varies 
from $\sin ^{2} i$ to 1 , respectively. Therefore, for inclinations about $60^{\circ}$, the corresponding value of $\sigma_{R}$ at a given point changes slightly and remains within 1-1.25 values of $\sigma_{\text {los,min }}$. This constraint does not take into account the data along the major axis, but can be checked independently using the gas rotation curve $v_{\mathrm{c}}$. We performed such a test for the galaxy NGC 338 .

NGC 338 is the only one in the Table 1 for which the gas rotation data are good enough. These data were obtained from the analysis of $\mathrm{H}_{\beta}$ and [OIII] emission lines and are described in detail in Zasov et al. (2012).

We used the equation (4) with known photometry and a set of $\sigma_{R, 0}$ values in a fixed range to determine the value of AD. This value should then be added to the average azimuthal velocity of stars to obtain the model profile $v_{\mathrm{c}}$ that should be compared with the observed rotation curve. After going through all the possible values of $\sigma_{R, 0}$ and calculating for them $\chi^{2}$ error we managed to find the optimal rotation curve that minimizes the difference between the gas data and the model. It should be noticed that for large inclination we need to account for the line-of-sight integration of the DF when determining rotation velocities. We did not make any reduction of data because for $i<60^{\circ}$ the distortion of the rotation curve for $R>h$ is minimal (Stepanova \& Volkov 2013).

Let us specify how to calculate the expression in brackets in the equation (4). The ratio $\frac{\sigma_{\varphi}^{2}}{\sigma_{R}^{2}}$ is known and equal to $f$. To calculate the logarithmic derivative $\frac{\partial \ln \Sigma_{\mathrm{s}}}{\partial \ln R}$ we assume that the mass-to-light ratio is constant along the disc. If so, instead of the surface density $\Sigma_{\mathrm{s}}$ we can use the stellar surface brightness of the disc measured in the bands tracing the old stellar population. For an exponential profile of brightness, this means that the logarithmic derivative should be replaced by $-\frac{R}{h}$, where $h$ denotes the disc scale length. The required photometry in the $I$ band was taken from Noordermeer \& van der Hulst (2007), where the disc scale length was found to be equal to $12.9^{\prime \prime}$. Finally, the last logarithmic derivative $\frac{\partial \ln \sigma_{R}^{2}}{\partial \ln R}$ can be numerically found from the fit of $\sigma_{\text {los,min }}(R)$ profile because $\sigma_{\text {los,min }}(R)$ is proportional to $\sigma_{R}(R)$ with the constant of proportionality $\sin ^{2} i+\alpha^{2} \cos ^{2} i$.

Results for NGC 338 are shown in Fig. 11. The figure demonstrates the gas and the stellar rotation curves, the model curve and the errors depending on the model parameter $\sigma_{R, 0}$. The $\chi^{2}$ curve demonstrates a strict global minimum at about $107 \mathrm{~km} / \mathrm{s}$. The optimal model for $v_{\mathrm{c}}$ is shown by dashed line and the shaded area corresponds to models with $\sigma_{R, 0}$ values differing from the optimal ones by less than $30 \mathrm{~km} / \mathrm{s}$. Notice that for $R \geq 27$ " the derivative of $\sigma_{\text {los,min }}$ produces large uncertainties because of a small number of points in this area and the AD is poorly defined. The gas rotation curve is approximated quite well within the radial region where model is good except areas close to the centre of the galaxy.

For NGC 338, the value of $\sigma_{\mathrm{los}, \min }\left(r_{\mathrm{e}, \mathrm{b}}\right)$ is close to $100 \mathrm{~km} / \mathrm{s}$ and hence the range of possible $\sigma_{R, 0}$ values vary from 100 till $125 \mathrm{~km} / \mathrm{s}$ as stated above. These constraints agree well with the values obtained from the $\mathrm{AD}$ analysis. Another less significant evidence comes from the mutual $\chi^{2}$-map, calculated for both axes simultaneously in the way described in Section 4. This map is shown in Fig. 3 and demonstrates that the best fit for two observational profiles $\sigma_{\text {los,maj }}$ and $\sigma_{\text {los,min }}$ is obtained for values of $\sigma_{R, 0}$ from the same range as above.

Thus, for galaxies with a large inclination, we can obtain constraints on the stellar radial velocity dispersion. These

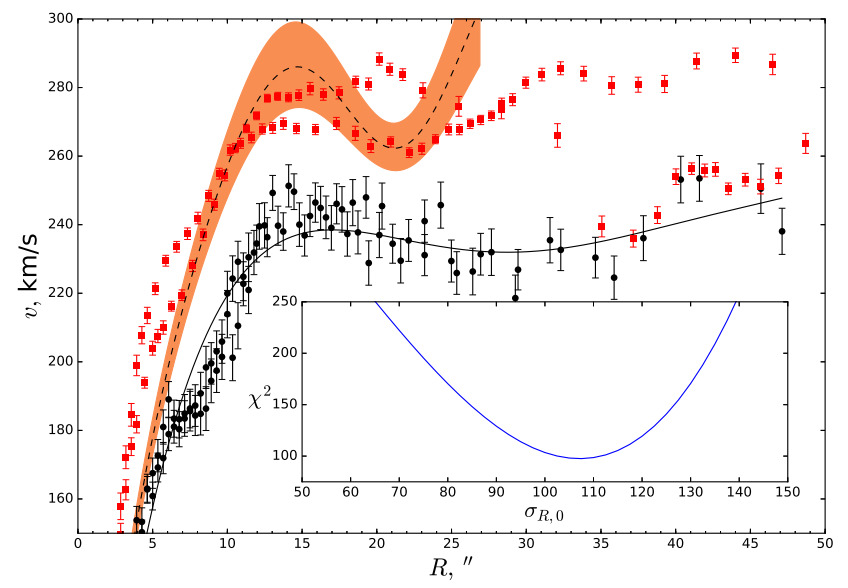

Figure 11. Reconstructed gas rotation curve of NGC 338. Filled black circles and solid line denote observational points and the best-fitting approximation, respectively. Red squares shows ionized gas rotation velocities, the so-called cold rotation curve. The subplot shows $\chi^{2}$ of gas model due to the AD prediction for different values of $\sigma_{R, 0}$. Gas rotation curve for $\sigma_{R, 0}=107 \mathrm{~km} / \mathrm{s}$ corresponding to the minimum of $\chi^{2}$ error is shown by the dashed line. The shaded area corresponds to the model gas rotation curves reconstructed by using values of $\sigma_{R, 0}$ that differ by no more than $30 \mathrm{~km} / \mathrm{s}$ from the optimal value.

constraints produce a narrow range of possible values. Example of NGC 338 shows that this range is consistent with the AD arguments. The value of $\sigma_{R}$ with the rotation curve of the galaxy and the mass model of its disc are sufficient to find the value of the parameter $Q$ and determine the dynamical status of the stellar disc.

\section{SUMMARY}

Dynamical models of galaxies require knowledge of the spatial stellar velocities distribution or at least of three components of SVE. The velocity dispersion in disc galaxies offers a clue for determining the dynamical status of a disc, its stability, dynamical history and relaxation processes. Unfortunately, velocities of stars in external galaxies are measured only in the projection on the line of sight. Full three-dimensional picture of the velocity distribution could be restored from the stellar spectral data indirectly. To reconstruct it several methods were proposed: via the exponential parametrization of the velocity dispersion profiles and subsequent data fitting along the major and minor axes (Gerssen et al. 1997, 2000; Shapiro et al. 2003; Gerssen \& Shapiro Griffin 2012); by apply the AD equation (Noordermeer et al. 2008; Sil'Chenko et al. 2011); through building a set of $N$-body marginally stable discs to choose the model that approximates spectral data in the best way (Zasov et al. 2008).

In this paper, we present a modification that does not employ a strict parametrization of the velocity dispersion profiles and requires only one additional assumption about the ratio $\sigma_{z} / \sigma_{R}=\alpha$, namely $\alpha$ remains constant along the whole profile or along its parts. This assumption allows us to introduce the proportionality between $\sigma_{\text {los,min }}$ and the velocity dispersion in the radial direction $\sigma_{R}$. Using this relationship, we can find the optimal value of $\alpha$ and the radial velocity dispersion normalization parameter $\sigma_{R, 0}$ by applying error minimization procedure simultaneously to the line-of-sight stellar velocity dispersion profiles along the major and minor axes of the galaxy which were pre-fitted. 
Our approach has been successfully tested on the galaxy NGC 1068 and showed the results similar to those obtained earlier by Shapiro et al. (2003). For further analysis, we chose three lenticular S0 galaxies NGC 1167, NGC 3245, NGC 4150 and one Sab galaxy NGC 338 with known rotation curves of stars and gas as well as the stellar velocity dispersion profiles.

Our results for these galaxies demonstrate a strong degeneracy trend for both major and minor axes. The simultaneous data consideration along two axes does not allow us to find optimal values of parameters in the physically meaningful range for galaxies with large inclinations (NGC 338, NGC 3245 and NGC 4150). In all cases, there is a gradual growth of $\chi^{2}$ with $\alpha$ increasing. For the galaxy at a moderate inclination NGC 1167, as well as for the galaxy NGC 1068, we managed to find the minimum of $\chi^{2}$. The results were verified by Monte Carlo method.

It should be noted that the best way to restore the SVE components for NGC 1167 galaxy is to reject the assumption $\sigma_{z} / \sigma_{R}=$ const throughout the whole profile and to divide the profile into the inner and outer parts, along which the main assumption still holds. For each of these parts we found the global minimum of $\chi^{2}$. The optimal values of $\alpha$ were found to be equal to 0.7 for the inner part and around 0.3 for the outer part. The decrease in $\alpha$ with rising distance from the centre is consistent with the results of the $N$-body simulations for this galaxy implemented by Zasov et al. (2008). Other numerical simulations also support such a result (see, e.g. Minchev et al. 2012). Thus, for the first time, the gradient in the meridional shape of the SVE was measured from the the spectral data. Stability of the results was verified using the Monte Carlo simulation. Recovered line-of-sight profiles of the stellar velocity dispersions for the major and minor axes showed good agreement with the observational data. Also, the resulting values of $\alpha$ are consistent with the disc dynamical heating theory. NGC 1167 has a moderate amount of gas in the molecular form $\left(M_{\mathrm{H}_{2}}=3.3 \times 10^{8} M_{\odot}\right)$ within $7.8 \mathrm{kpc}$, or $25^{\prime \prime}\left(\mathrm{O}^{\prime}\right.$ Sullivan et al. 2015). Molecular gas is usually thought as a 'three-dimensional scattering agent' that increases the vertical velocity dispersion above the level of the bending instability. At the same time, the outer parts of a disc may remain 'cold' just at the level $\sigma_{z} / \sigma_{R} \approx 0.3$ (Rodionov \& Sotnikova 2013).

For galaxies with high inclinations SVE was not reconstructed because contribution of the vertical velocity dispersion component in the line-of-sight velocity dispersion data is comparable with observational uncertainties for such galaxies and thus cannot be extracted from the data in practice. As a result, the formal optimal value of $\alpha$ falls on one of the boundaries of the range of $\alpha$ (either $\alpha$ minimal or equal to 1 ). Thus, the result for the galaxy NGC 2775 from the paper Gerssen \& Shapiro Griffin (2012), where $\alpha \approx 1$ was obtained, can be considered as a formal solution.

Despite the fact that the impact of a high inclination does not allow us to restore correctly all velocity dispersion components, for these galaxies we can constrain the possible values of the radial velocity dispersion $\sigma_{R}$ by a sufficiently narrow range. For example, for NGC 338, for which there are good enough gas rotation curve data, these constraints were verified with the help of the AD equation. Obtained values were in good agreement with each other and with the values from the $\chi^{2}$-maps.

\section{ACKNOWLEDGEMENTS}

We are grateful to Alexei Moiseev and Ivan Katkov for providing spectral data. We thank Saint Petersburg State University research grant 6.38.335.2016 (AAM) and grant of the Russian Foundation for Basic Research number 14-02-810 (NYS) for partial support. We thank the anonymous referee for his/her thorough review and highly appreciate the comments and suggestions that significantly contributed to improving the quality of the article.

This research makes use of the NASA/IPAC Extragalactic Database (NED) which is operated by the Jet Propulsion Laboratory, California Institute of Technology, under contract with the National Aeronautics and Space Administration, and the LEDA database (http://leda.univ-lyon1.fr).

\section{REFERENCES}

Afanasiev V. L., Moiseev A. V., 2005, Astronomy Letters, 31, 194

Binney J., Tremaine S., 2008, Galactic Dynamics: Second Edition. Princeton University Press

Bottema R., 1993, A\&A, 275, 16

Bottema R., Gerritsen J. P. E., 1997, MNRAS, 290, 585

Bovy J., Rix H.-W., 2013, ApJ, 779, 115

Capuzzo-Dolcetta R., Leccese L., Merritt D., Vicari A., 2007, ApJ, 666, 165

Dehnen W., Binney J. J., 1998, MNRAS, 298, 387

Di Teodoro E. M., Fraternali F., 2015, MNRAS, 451, 3021

Dorman C. E., et al., 2015, ApJ, 803, 24

Fabricius M. H., Saglia R. P., Fisher D. B., Drory N., Bender R., Hopp U., 2012, ApJ, 754, 67

Fisher D. B., Drory N., 2010, ApJ, 716, 942

Gerssen J., Shapiro Griffin K., 2012, MNRAS, 423, 2726

Gerssen J., Kuijken K., Merrifield M. R., 1997, MNRAS, 288, 618

Gerssen J., Kuijken K., Merrifield M. R., 2000, MNRAS, 317, 545

Kregel M., van der Kruit P. C., Freeman K. C., 2005, MNRAS, 358, 503

Lacey C. G., 1984, MNRAS, 208, 687

Le Borgne D., Rocca-Volmerange B., Prugniel P., Lançon A., Fioc M., Soubiran C., 2004, A\&A, 425, 881

Martinsson T. P. K., Verheijen M. A. W., Westfall K. B., Bershady M. A., Schechtman-Rook A., Andersen D. R., Swaters R. A., 2013, A\&A, 557, A 130

Minchev I., Famaey B., Quillen A. C., Dehnen W., Martig M., Siebert A., 2012, A\&A, 548, A127

Moiseev A. V., 2001, Bulletin of the Special Astrophysics Observatory, 51, 11

Noordermeer E., van der Hulst J. M., 2007, MNRAS, 376, 1480

Noordermeer E., van der Hulst J. M., Sancisi R., Swaters R. A., van Albada T. S., 2005, A\&A, 442, 137

Noordermeer E., van der Hulst J. M., Sancisi R., Swaters R. S., van Albada T. S., 2007, MNRAS, 376, 1513

Noordermeer E., Merrifield M. R., Aragón-Salamanca A., 2008, MNRAS, 388,1381

O’Sullivan E., Combes F., Hamer S., Salomé P., Babul A., Raychaudhury S., 2015, A\&A, 573, A111

Rodionov S. A., Sotnikova N. Y., 2006, Astronomy Reports, 50, 983

Rodionov S. A., Sotnikova N. Y., 2013, MNRAS, 434, 2373

Rodionov S. A., Athanassoula E., Sotnikova N. Y., 2009, MNRAS, 392, 904

Schwarzschild M., 1979, ApJ, 232, 236

Shapiro K. L., Gerssen J., van der Marel R. P., 2003, AJ, 126, 2707

Sil'Chenko O. K., Chilingarian I. V., Sotnikova N. Y., Afanasiev V. L., 2011, MNRAS, 414, 3645

Spitzer Jr. L., 1942, ApJ, 95, 329

Stepanova K. V., Volkov E. V., 2013, Astronomy Letters, 39, 405

Syer D., Tremaine S., 1996, MNRAS, 282, 223

Toomre A., 1964, ApJ, 139, 1217

Verheijen M. A. W., Bershady M. A., Andersen D. R., Swaters R. A., Westfall K., Kelz A., Roth M. M., 2004, Astronomische Nachrichten, 325,151

Zasov A. V., Khoperskov A. V., 2003, Astronomy Letters, 29, 437 
Zasov A. V., Moiseev A. V., Khoperskov A. V., Sidorova E. A., 2008, Astronomy Reports, 52, 79

Zasov A. V., Khoperskov A. V., Katkov I. Y., Afanasiev V. L., Kaisin S. S., 2012, Astrophysical Bulletin, 67, 362

de Lorenzi F., Debattista V. P., Gerhard O., Sambhus N., 2007, MNRAS, 376,71

de Lorenzi F., Gerhard O., Saglia R. P., Sambhus N., Debattista V. P., Pannella M., Méndez R. H., 2008, MNRAS, 385, 1729

van den Bosch R. C. E., van de Ven G., Verolme E. K., Cappellari M., de Zeeuw P. T., 2008, MNRAS, 385, 647

van der Kruit P. C., Freeman K. C., 1986, ApJ, 303, 556

van der Kruit P. C., Searle L., 1981, A\&A, 95, 105 